\title{
ACTIVIDAD DE BÚSQUEDAS EN INTERNET COMO VARIABLE PARA DETERMINAR LA AFLUENCIA A MUSEOS
}

\author{
Raúl Gómez Martínez \\ Beatriz Rodríguez Herráez \\ Diana Catalina Pérez-Bustamante Yábar \\ Universidad Rey Juan Carlos. Madrid
}

\section{RESUMEN}

Tanto Internet como las nuevas tecnologías tienen cada vez más importancia en las investigaciones académicas o profesionales que se realizan. Con este trabajo tratamos de analizar si las estadísticas de búsqueda en un buscador como Google tienen capacidad de predicción respecto al número visitantes que van a acudir a un museo. Se trata de demostrar científicamente a través de un modelo econométrico que las estadísticas de búsqueda en Google sirven para conocer la posibilidad de visitantes de un museo. Con este artículo se pretenden realizar nuevas aportaciones a la gestión de los museos y sugerir la utilización de datos existentes que permitan conocer con cierta antelación la afluencia de público que se tendrá y así poder desarrollar estrategias.

Palabras clave: Visitantes de museos, expectativas, Google Trends, turismo cultural, Internet.

\section{Activity of searches in Internet like variable for deteminar the visitors to museums}

\section{ABSTRACT}

Internet and new technologies have become increasingly important in academic or professional research. In this paper we analyze whether Internet search activity on Google

Recibido: 10 de marzo de 2015

Devuelto para su revisión: 23 de septiembre de 2015

Aceptado: 20 de noviembre de 2015

Departamento de Economía de la Empresa. Facultad de Ciencias Jurídicas y Sociales. Universidad Rey Juan Carlos. Paseo Artilleros, s/n. 28032 MADRID (España).

E-mail: raul.gomez.martinez@urjc.es, beatriz.rodriguez@urjc.es, diana.perezbustamante@urjc.es 
have predictive power for the number of visitors a museum will receive. It is scientifically proven through an econometric model that Google search statistics used to determine the possibility of a museum visitor. This article aims to provide new contributions to the management of museums and suggest the use of existing data to determine the number of visitors of the museum so we can develop strategies.

Keywords: Museum visitors, expectations, Google Trends, cultural tourism, Internet.

\section{INTRODUCCIÓN}

Desde hace muchos años el turismo ha sido uno de los pilares económicos de España y ésta situación se mantiene a pesar de la crisis que arrastramos desde hace ya más de un lustro. En el pasado año 2013, España fue el tercer destino internacional de turistas alcanzando los 60,7 millones (IET, 2014a), aumentando un 5,6 por ciento respecto al año anterior. Además, se ha consolidado la tendencia que comenzó el pasado año y que rompió con la que existía desde hace varios años antes, se ha mantenido el ascenso en el gasto real medio diario realizado por los turistas internacionales que se ha situado en los 109 euros (EIT, 2014b).

Sin embargo, es indiscutible que el sector turístico se encuentra desde hace años con importantes debilidades tanto de naturaleza estructural como competitiva, fundamentalmente en la oferta del turismo de sol y playa. La aparición de destinos competidores fuertes y los problemas de concentración geográfica y estacionalidad han sido las causas de este deterioro.

Para tratar de solventar estas deficiencias y estando demostrada la importancia del turismo en la economía española y más en tiempos difíciles donde casi el único sector que aporta datos positivos es el turístico, las instituciones públicas tratan de establecer proyectos que impulsen el turismo, un claro ejemplo es el desarrollado por el Ministerio de Industria, Turismo y Comercio bajo el nombre de «Plan del Turismo Español Horizonte 2020» (SGT, 2008). Con estos proyectos se trata de analizar la situación actual del sector desde el punto de vista de sus recursos y capacidades, así como desde las fortalezas y debilidades del mismo.

Las autoridades competentes turísticas españolas están tratando de potenciar otros tipos de turismo complementarios al tradicional de sol y playa, y para ello han desarrollado una serie de estrategias y acciones. Un tipo de turismo que presenta unas características favorables es el turismo cultural, entre las razones que justifican ésta afirmación se encuentra el consumo que se realiza y que es continuado a lo largo del año y no se centra en los meses estivales (Ortega y Rodríguez, 2008), además el gasto medio de los turistas es bastante más elevado que el gasto medio del resto de turistas que nos visitan (IET, 2012) y por último este turismo permite proyectar una imagen positiva de España en cuanto a la cantidad de recursos culturales con los que cuenta.

Entre los recursos culturales estos pueden tener naturaleza material o inmaterial, si bien son más fáciles de identificar y cuantificar los primeros de ellos. Las manifestaciones que se incluyen en las de tipo cultural pueden ser numerosas incluyendo desde los monumen- 
tos, la gastronomía, la artesanía, los monasterios o el arte hasta los museos. Estos últimos, los museos, se pueden considerar centros donde se recogen obras de arte para exponerlas, restaurarlas y conservarlas, y que sirve como un medio de comunicación capaz de alcanzar a un gran número de público, entre ellos turistas y residentes (ICOM, 1994).

En la Comunidad de Madrid, el sector de la cultura es un foco de atención y tiene un gran peso dentro de la actividad económica. Los museos son una parte importante dentro de este sector pero hay que tener en cuenta que un buen número de ellos no reúnen las condiciones necesarias para ser visitados y considerarse productos turísticos. La oferta de museos de la Comunidad de Madrid asciende a más de 90 (Ministerio de Educación, Cultura y Deporte, 2013) y se agrupan en diferentes tipologías según su contenido, pudiendo encontrar museos de Arte, de Arqueología e Historia, otros de Ciencias y Tecnología, o de Ciencias Naturales, de Antropología y Etnografía, incluso otros tipos de museos especializados o regionales. Dentro de la Comunidad de Madrid el mayor número de su patrimonio se concentra en la ciudad de Madrid, y más concretamente en el conocido Paseo del Arte que está delimitado por el Museo del Prado, el Museo Nacional Central de Arte Reina Sofía y el Museo Thyssen-Bornemisza.

El número de visitantes que acuden a los principales museos va incrementándose anualmente a pesar de los factores del entorno y como consecuencia de las excelentes colecciones que poseen y de las exposiciones que presentan temporalmente estos museos. En concreto, el pasado año 2013 los museos nacionales que dependen de la Subdirección General de Museos Estatales obtuvieron un ascenso en el número de visitantes de 4,9 por ciento respecto al año 2012, lo que supone en términos absolutos un incremento de 89.297 visitantes y sitúa el número de visitas de estos museos en 1.927.250 (Ministerio de Educación, Cultura y Deporte, 2014)

\section{2. ¿CÓMO DECIDIR QUÉ MUSEO VISITAR?}

Los museos madrileños son, en general, poco conocidos y por ende poco visitados, a excepción de tres casos muy concretos. Resultan interesantes las visitas que reciben los tres principales museos, El Prado, Reina Sofía y Thyssen. Entre ellos, es el museo del Prado el que más visitas recibe, en el año 2012 contó con 2.710 .906 visitantes (IET, 2014c) lo que supuso descenso del 3,9 por ciento respecto al año anterior. El museo Reina Sofía en ese mismo año superó los 2,7 millones de visitantes lo que representó un $10 \%$ más que el año anterior. El museo Thyssen batió su record de visitantes superando la barrera del millón, lo que significó un 30\% más que en el 2010.

La procedencia de los visitantes de los museos es muy variada, aunque el perfil del visitante medio se corresponde con una mujer de edad media 42 años, con estudios universitarios, que se encuentra en una situación laboral activa y que reside en España (Laboratorio Permanente de Público de Museos, 2011).

Nos estamos refiriendo a los visitantes de los museos, pero para los objetivos de nuestro trabajo nos deberíamos plantear si se trata en realidad de visitantes o de usuarios. En nuestra opinión y coincidiendo con García Blanco (2002) nos parecen dos términos que no deben tratarse como incompatibles, sino que por el contrario están estrechamente relacionados. Es cierto que cada término tiene unas connotaciones diferentes, cuando nos 
referimos al usuario de los museos estamos incluyendo a los visitantes. En el caso que nos ocupa, el término de usuario resulta como consecuencia de considerar el museo como un organismo que ofrece una serie de servicios y cuando una persona hace uso de ellos actúa como un usuario. Siguiendo en esta misma línea convendría señalar en este punto que el concepto de usuario es idéntico al de consumidor, razón por la cual podríamos plantearnos la manera en que los usuarios/consumidores de los museos inician su comportamiento de compra.

El modelo de decisión del consumidor fue propuesto en su inicio por los profesores Engel, Kollat y Blackwell a los que se unió posteriormente Miniard. Su propuesta consistía en plantear teóricamente cómo los consumidores toman decisiones lógicas en base a unos hechos realizados con anterioridad, es decir, el modelo se fundamente en una secuencia de etapas por las que va pasando el consumidor y que van desde el reconocimiento de la necesidad y la búsqueda de información hasta la compra final (Figura 1).

\section{Figura 1}

\section{MODELO DE PROCESO DE DECISIÓN DE COMPRA}

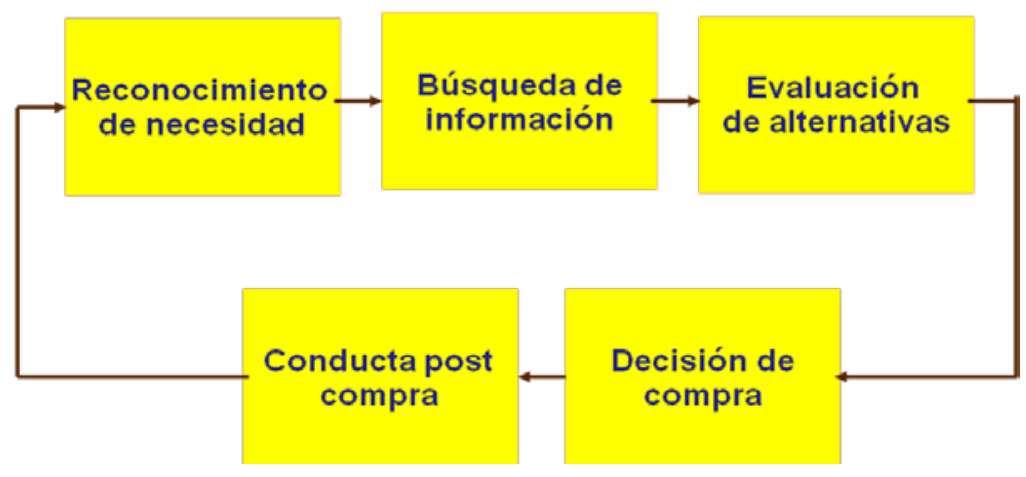

Fuente: Blackwell, Miniard y Engel (2005).

Los estudios sobre los usuarios de los museos, ya sean sobre las motivaciones de los visitantes, las experiencias de los visitantes o cuáles son las expectativas que tienen éstos, son importantes para las instituciones culturales ya que son necesarios en términos de gestión y desarrollo (Weil, 2000). Según el Comité de Investigación de Audiencia y Evaluación de la Asociación Americana de Museos, los estudios sobre visitantes utilizan un enfoque sistemático, donde se incluye un conocimiento relacionado con el visitante in-situ y también sobre los potenciales visitantes. Estos estudios se utilizan para ayudar a los museos a planificar y ejecutar las actividades públicas relacionadas de mejor manera (CARE, 2009).

Las necesidades que manifiestan los visitantes/usuarios de los museos suelen ser concretas, y están relacionadas con la identidad de las personas y con su estatus social (Ministerio de Cultura, 2012). En el estudio realizado por el Ministerio de Cultura para conocer a los visitantes de los museos, el motivo que se repite más frecuentemente para visitar el 
museo es el de la curiosidad, seguido de ocupar el tiempo de ocio. Diferentes estudios y autores como Packer y Ballantyne (2002) nos comentan que la parte correspondiente al aprendizaje y a la educación, es de especial relevancia a la hora de captar la atención del visitante de un museo. Este tipo de visitantes perciben el museo como un lugar que contiene mucha información presentada de una manera interesante y en donde el visitante está dispuesto a esforzarse en aprender lo que se les ofrece, obteniendo una satisfacción una vez realizado ese esfuerzo. En líneas generales, se observa que uno de los motivos principales está directamente relacionado con el nivel de educación de los usuarios, como contemplamos en muchas observaciones realizadas por numerosos autores como Schuster (2001) y DiMaggio (1978); ambos analizando a la población estadounidense, pero también ha estudiado para otro países como Alemania, Países Bajos (Ganzeboom, 1982), Francia, Polonia y Grecia (Bourdieu y Darbel, 1990), Suecia y Gran Bretaña (Schuster, 1991) y Canadá (Schliewen, 1977).

En sentido totalmente contrario encontramos las aportaciones realizadas por Hood (1983) y Prentice, Davies y Beeho (1997), que mantenían la teoría de que para definir el perfil de los visitantes de los museos las variables sociodemográficas no son determinantes y aportan una escasa información sobre las motivaciones para la visita. Estos autores proponen como variable fundamental para estudiar las motivaciones de los visitantes sus características psicográficas.

Una vez reconocida la necesidad, la etapa siguiente que realiza el consumidor es la del buscar información y para ello procede inicialmente a un análisis interno de la información que posee haciendo uso de la memoria, para más tarde efectuar un análisis externo que incluye desde los familiares o amigos hasta medios masivos o below the line.

Esta etapa de búsqueda de información variará de unos visitantes a otros y dependerá de algunos factores como pueden ser la motivación más o menos fuerte que se tenga en el producto/servicio, la cantidad de información previa que se tenga, la facilidad de obtener información o el valor que se le asigne a la información (Kotler, Bowen y Makens, 2004).

Las estrategias de búsqueda de información a través de las fuentes externas no tienen porque ser uniformes para todos los visitantes y varían en función de circunstancias como la frecuencia de la visita, la compañía y el lugar de residencia (Ministerio de Cultura, 2012). Este tipo de información puede ser pasiva o activa. La búsqueda pasiva se produce cuando el visitante lo único que hace es poner más atención en la información que le será relevante para el proceso en el que se encuentra de toma de decisión. En otros casos, la búsqueda será activa y los visitantes recurrirán a fuentes de información donde encontrar lo que buscan. Las fuentes de información pueden clasificarse según sean personales o impersonales y sean controlables o no en: fuente personal y controlable; 2) fuente personal e incontrolable; 3) fuente impersonal y controlable; 4) fuente impersonal e incontrolable.

En el ámbito del sector turístico, el primer tipo se refiere a la información que se recibe por parte de las personas vinculadas a las diferentes organizaciones turísticas. En el segundo tipo se encuentran los canales de expertos del sector turístico, así como a través de los canales sociales, formados principalmente por líderes de opinión, escritores turísticos, familiares, amigos y vecinos (Rompf, 2002; Rompf y Ricci, 2005; Wang, Severt y Rompf, 2005). 
El tercer tipo se corresponde con la información que transmiten los medios masivos convencionales de comunicación, diarios, revistas, radio, televisión, cine, publicidad exterior e Internet; o a través de otros medios masivos.

El cuarto tipo es de naturaleza impersonal e incontrolable, identificándose con todos aquellos hechos y acontecimientos fortuitos que pueden ocurrir a lo largo del tiempo.

Según el estudio del Ministerio de Cultura la fuente de información más utilizada para visitar un museo es la recomendación de un familiar, un amigo o un conocido, seguido de la recomendación de algún compañero de trabajo o estudios y en tercer lugar la televisión. Las dos primeras fuentes se corresponden con fuentes personales e incontrolables por parte del museo que tienen una gran importancia pero sobre las que el museo no tiene ningún dominio. Entre los medios masivos y controlables Internet ocupa la tercera posición por detrás de la televisión y la radio, debiéndose tener en cuenta además que adquiere mayor importancia entre las personas que acuden con niños, los estudiantes y las personas que se encuentran en una posición laboral activa. Este medio de comunicación está incrementado su importancia anualmente aunque no lo ha hecho proporcionalmente al aumento que se ha producido en la utilización del mismo para la búsqueda de información de los museos.

Sin embargo, en el último lustro las pinacotecas más importantes a nivel internacional se han volcado en el uso de las nuevas tecnologías, incluido Internet, y permiten visitar tanto las exposiciones que cuelgan de sus paredes como las propias instalaciones aunque aún no han sido capaces de aprovechar todas las posibilidades que ofrece la tecnología y utilizan sus páginas Web como un tablón de anuncios donde informar de los aspectos más prácticos como el horario de visita, la accesibilidad, las exposiciones actuales, las actividades que se pueden realizar e incluso la venta anticipada de las entradas. Del estudio realizado por Socialwin (2013) que analiza la utilización de las redes sociales por parte de los museos, se deduce que cada vez son más los que tienen perfil en Facebook y Twitter, en concreto los museos que usan Facebook asciende a un 63 por ciento, mientras que los que usan Twitter son muchos menos y representan el 37 por ciento. Sin embargo, esta presencia no está directamente relacionada con la utilización que hacen de ellas y en un elevado número de casos utilizan estas herramientas tan solo para colgar información pero desaprovechan las posibilidades de interactuación que permiten o las posibles relaciones que se pudieran crear.

\section{ACTIVIDAD DE BÚSQUEDAS EN INTERNET COMO VARIABLE EXPLICA- TIVA EN ECONOMÍA Y EMPRESA}

Con el desarrollo de la tecnología y el boom de las redes sociales experimentado en la segunda década del siglo XXI, están surgiendo aproximaciones alternativas al uso de encuestas que nos facilitan el estudio sobre cómo los usuarios y consumidores buscan información y evalúan alternativas antes de sus decisiones de compra o de inversión. Este nuevo enfoque se manifiesta en nuevas técnicas y experimentos para estudiar y evaluar el estado de ánimo de inversores y consumidores.

El R-Word Index del semanario The Economist mide el número de artículos publicados en periódicos como Financial Times o The Wall Street Journal, en los que se utiliza la palabra «recesión». Aaron Gerow y Mark Keane (2011) estudian la relación entre la 
frecuencia de uso de diferentes palabras y la evolución de los mercados. Estos autores proponen que cambios en la distribución de la frecuencia en la que se usan determinadas palabras en prensa reflejan los movimientos del mercado de valores. Bollen et al (2010) manifiestan que este principio es el seguido por los gestores del fondo de inversión Derwent Absolute Return Fund Ltd para establecer sus decisiones de inversión. También en el ámbito financiero está cobrando especial interés en la investigación la actividad de búsquedas en Internet medida a través de Google Trends y las estadísticas de búsquedas en internet que facilita. Gomez-Martínez (2011) elaboran un modelo en el que la evolución de los índices bursátiles queda explicada por el estado de ánimo de los inversores considerando este estado de ánimo según las estadísticas de búsquedas en Google de determinados términos relacionados con los mercados financieros. A partir de una muestra de datos semanales que abarca desde Enero de 2006 hasta Diciembre de 2010, el modelo estimado presenta una elevada capacidad explicativa según los coeficientes de determinación $\mathrm{R}^{2}$ obtenidos y se aprecia que términos pesimistas como «Economic Crisis» o «Bear Market» son altamente significativos y presentan una relación negativa con respecto a la evolución del mercado. Gómez-Martínez (2012) trabaja este principio proponiendo un modelo de inversión basado en un índice de aversión al riesgo que se desarrolla a partir de estas búsquedas en Internet. Entendemos que esta herramienta si es válida para explicar el sentimiento del inversor puede ser igual de potente a la hora de explicar el proceso de selección del consumidor.

Otros trabajos publicados con posterioridad también utilizan la información de Google Trends para explicar la demanda y volatilidad grandes valores cotizados en el NYSE y en el NASDAQ (Vlastakis et al, 2012), mientras que Smith (2012) la utiliza para explicar la volatilidad del mercado de divisas. Rose et al (2012) utilizan la información de Google Trends para explicar la iliquidez del dólar durante la crisis financiera. En un plano económico no estrictamente financiero, Askitas y Zimmermann (2009) demostraron la fuerte correlación de búsquedas de términos en alemán como «oficina de desempleo» o «tasa de desempleo» con la evolución del mercado laboral alemán, estudio similar al de McLaren y Shanbhoge (2011) que en el ámbito del mercado británico usaron el volumen de estadísticas de Google para explicar la evolución del mercado laboral e inmobiliario.

Teniendo en cuenta los antecedentes del estudio descritos, podemos asumir que las decisiones de inversión están basadas en las expectativas futuras de los inversores y que estas expectativas están condicionadas por el estado de ánimo de los inversores que les hacen ser más o menos aversos al riesgo. El problema al que nos enfrentamos es cómo medir este estado de ánimo de los inversores y, por lo tanto, su grado aversión al riesgo.

A modo ilustrativo de la capacidad predictiva de la actividad de búsqueda en Internet, hicimos el siguiente ejercicio en las pasadas elecciones generales españolas del 20 de Noviembre de 2011, donde hicimos un simple ejercicio descargando las estadísticas de búsquedas de las siglas los partidos políticos con representación en el congreso restringiendo la zona al periodo de la campaña electoral y a la zona geográfica de España. Los promedios de los índices de búsquedas realizadas se recogen en la Tabla 1, y sobre ese promedio hacemos una simple regla de tres para calcular el porcentaje de los votos que recibiría cada partido. 
Tabla 1

ELECCIONES 20 NOV 2011

\begin{tabular}{|l|r|r|r|r|r|r|r|r|r|}
\hline & PP & PSOE & \multicolumn{1}{c|}{ IU } & UPyD & CIU & PNV & ERC & BNG & TOTAL \\
\hline PROMEDIO & 56 & 35 & 11 & 18 & 4 & 3 & 3 & 2 & 133 \\
\hline$\%$ VOTOS & $42 \%$ & $26 \%$ & $8 \%$ & $14 \%$ & $3 \%$ & $2 \%$ & $2 \%$ & $2 \%$ & $100 \%$ \\
\hline
\end{tabular}

Fuente: elaboración propia.

El ejercicio lo hicimos en la jornada de reflexión no nos llevó más de 5 minutos, fue gratuito y la verdad es que a excepción de UPyD que generó más interés que votos, poco nos equivocamos como se puede observar en la Tabla 2. Graefe et al (2010) o Lui et al (2011) han publicado artículos que abundan en la utilidad de Google Trends a la hora de predecir resultados electorales.

Tabla 2

RESULTADOS ELECCIONES 20 NOV 2011

\begin{tabular}{|l|r|r|}
\hline & Previsión & Real \\
\hline PP & $42 \%$ & $45 \%$ \\
\hline PSOE & $26 \%$ & $29 \%$ \\
\hline IU & $8 \%$ & $7 \%$ \\
\hline UPyD & $14 \%$ & $5 \%$ \\
\hline CIU & $3 \%$ & $4 \%$ \\
\hline PNV & $2 \%$ & $1 \%$ \\
\hline ERC & $2 \%$ & $1 \%$ \\
\hline BNG & $2 \%$ & $1 \%$ \\
\hline AMAIUR & & $1 \%$ \\
\hline
\end{tabular}

Este modelo ha sido desarrollado por Gómez-Martínez y Prado (2013) demostrando a través de un modelo econométrico de datos de panel que las búsquedas en Google de las siglas de los partidos políticos en el mes de unas elecciones puede explicar más del 70\% del porcentaje de voto sobre la población total.

Por lo tanto, si la actividad de búsqueda en Internet tiene la capacidad explicativa y descriptiva que proponen los estudios anteriormente citados, ¿por qué no utilizarla para explicar la afluencia de visitantes a museos?

\section{HIPÓTESIS Y METODOLOGÍA}

En el siguiente análisis pretendemos estudiar si el interés por los museos se traduce en una mayor afluencia de visitantes. Para ello asumimos que el nivel de popularidad de un museo y el grado de interés que este despierta puede ser medido a través de las estadísticas de búsqueda realizadas en Google realizadas con el nombre del museo. 
Por lo tanto las hipótesis a validar en el estudio serían:

- H1: Las series históricas de estadísticas de búsquedas en Google son una variable con capacidad para explicar los niveles de asistencia a una institución cultural, en concreto a un museo.

- H2: Un mayor interés por un museo implica un mayor volumen de visitantes.

Nuestra metodología de estudio será proponer un sencillo modelo econométrico en el que la variable explicativa serán las estadísticas de búsquedas realizadas en Google para cada museo, y como variable explicada el volumen total de visitantes.

$$
Y_{i t}=\alpha_{i}+\beta_{i t} X_{i t}+\varepsilon_{t}
$$

Donde:

$Y_{i t}$ Es el volumen total de asistentes al museo «i» en el periodo «t».

$X_{i t}$ Es el nivel de estadísticas de búsquedas de Google para el nombre del museo «i» en el periodo «t»

$\alpha_{i}$ Es el término contante

$\beta_{i t}$ Es el coeficiente de la regresión

$\varepsilon_{t} \quad$ Es el término de error

Validaremos por tanto la hipótesis H1 si los coeficientes $\beta$ calculados en las regresiones resultan significativamente distintos de cero para un nivel de confianza del 99\% ó 95\%. Así mismo daremos por válida la hipótesis H2 si los coeficientes $\beta$ calculados son positivos lo que implicaría que un mayor interés por el museo se corresponde con un mayor volumen de visitantes.

\section{DATOS}

Disponemos de datos de series temporales de visitantes al Museo de Arte Reina Sofía de frecuencia semanal, los cuales fueron facilitados directamente por los responsables de gestionar dicho museo. Disponemos también de datos del Museo del Prado de frecuencia semanal, también facilitados por el propio museo. Por último disponemos de los datos de asistencia mensual a los museos gestionados por el Ministerio de Cultura. Estos datos se han descargado directamente de la web de «Cifras de visitantes de los Museos Estatales» ${ }^{1}$.

El conjunto de museos a estudiar en un origen era:

- Museo de Arte Reina Sofía

- Museo del Prado

1 Las cifras de asistentes a estos museos se pueden descargar del siguiente link:

http://www.mcu.es/visitantemuseo/cargarFiltroBusqueda.do?layout=visitantemuseo\&cache=init\&language=es 
- Museo Arqueológico Nacional

- Museo Casa de Cervantes

- Museo Cerralbo

- Museo de Altamira

- Museo de América

- Museo del Greco

- Museo del Traje

- Museo Nacional de Antropología

- Museo Nacional de Arqueología Subacuática. ARQUA

- Museo Nacional de Arte Romano

- Museo Nacional de Artes Decorativas

- Museo Nacional de Cerámica

- Museo Nacional de Escultura

- Museo Nacional de Reproducciones Artísticas

- Museo Nacional del Romanticismo

- Museo Romántico

- Museo Sefardí

- Museo Sorolla

Para estos museos se descargaron las estadísticas de Búsquedas de Google introduciendo el nombre de cada museo en la herramienta Google Trends ${ }^{2}$. Observamos que Google no ofrece estadísticas para los museos menos conocidos y cuyo volumen de estadísticas es pequeño por lo que tuvimos que restringir el análisis a aquellos museos para los cuales el volumen de búsquedas en Google era relevante y disponíamos datos de ello. Los museos finalmente analizados son:

- Museo de Arte Reina Sofía

- Museo del Prado

- Museo de Altamira

- Museo Arqueológico Nacional

- Museo Casa de América

- Museo Casa Cervantes

- Museo del Greco

- Museo Nacional de Escultura

Estos museos presentan niveles de asistencia muy diferentes como se puedo observar en la figura 2 donde se muestra la media de asistencia por semana a cada museo.

2 Se puede acceder a la aplicación Google Trends a través del siguiente link: http://www.google.es/trends/ 
Figura 2

NIVEL DE ASISTENCIA MEDIA SEMANAL EN LOS MUSEOS

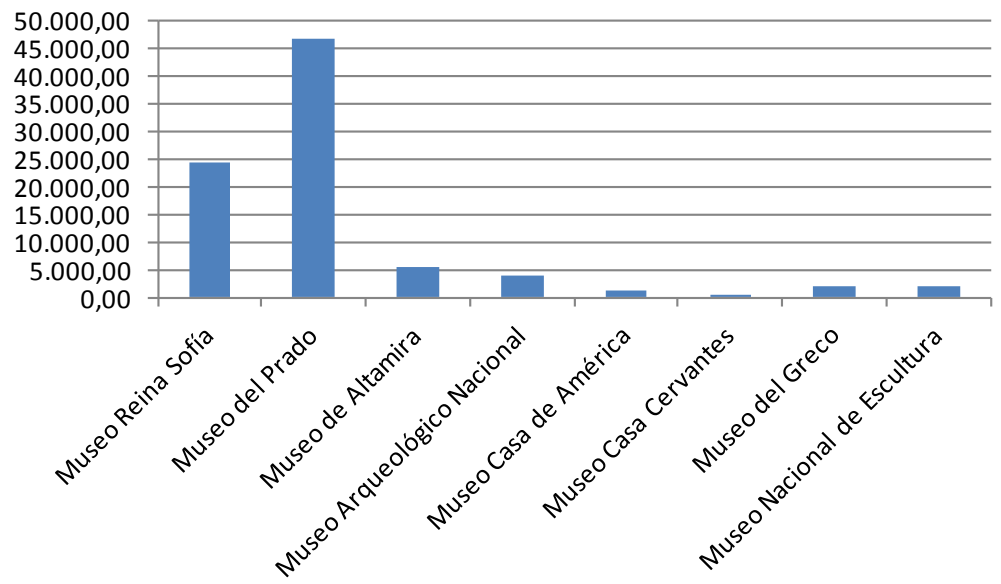

Los datos estadísticos de las series históricas de asistentes a museos están contenidos en la tabla 3.

Tabla 3

SERIES HISTÓRICAS DE ASISTENTES A MUSEOS

\begin{tabular}{|l|r|r|r|r|r|r|r|r|}
\hline & $\begin{array}{c}\text { Museo } \\
\text { Reina } \\
\text { Sofía }\end{array}$ & $\begin{array}{c}\text { Museo del } \\
\text { Prado }\end{array}$ & $\begin{array}{c}\text { Museo de } \\
\text { Altamira }\end{array}$ & $\begin{array}{c}\text { Museo } \\
\text { Arqueológico } \\
\text { Nacional }\end{array}$ & $\begin{array}{c}\text { Museo } \\
\text { Casa de } \\
\text { América }\end{array}$ & $\begin{array}{c}\text { Museo } \\
\text { Casa } \\
\text { Cervantes }\end{array}$ & $\begin{array}{c}\text { Museo } \\
\text { del } \\
\text { Greco }\end{array}$ & $\begin{array}{c}\text { Museo } \\
\text { Nacional } \\
\text { de } \\
\text { Escultura }\end{array}$ \\
\hline Periodo analizado & $\begin{array}{r}2004 / 02 / 29 \\
2012 / 08 / 05\end{array}$ & $\begin{array}{c}2004 / 01 / 04 \\
2012 / 09 / 09\end{array}$ & $\begin{array}{c}2004: 01- \\
2012: 09\end{array}$ & $\begin{array}{c}2004: 01- \\
2011: 07\end{array}$ & $\begin{array}{c}2004: 01- \\
2012: 09\end{array}$ & $\begin{array}{c}2004: 01- \\
2012: 09\end{array}$ & $\begin{array}{c}2004: 01- \\
2012: 09\end{array}$ & $\begin{array}{c}2004: 01- \\
2012: 09\end{array}$ \\
\hline Frecuencia & Semanal & \multicolumn{1}{|c|}{ Semanal } & Mensual & Mensual & Mensual & Mensual & Mensual & Mensual \\
\hline Observaciones & 435,00 & 454,00 & 103,00 & 91,00 & 103,00 & 103,00 & 103,00 & 103,00 \\
\hline Media & $24.515,00$ & $46.958,00$ & $21.691,00$ & $16.105,00$ & $5.051,20$ & $1.880,40$ & $8.810,10$ & $8.629,40$ \\
\hline Mediana & $23.787,00$ & $47.137,00$ & $21.379,00$ & $14.878,00$ & $4.845,00$ & 0,00 & $2.838,00$ & $7.623,00$ \\
\hline Mínimo & $8.368,00$ & $14.028,00$ & $3.922,00$ & $1.456,00$ & $1.945,00$ & 0,00 & 0,00 & $2.958,00$ \\
\hline Máximo & $51.696,00$ & $115.460,00$ & $53.736,00$ & $45.512,00$ & $10.036,00$ & $7.308,00$ & $29.281,00$ & $20.585,00$ \\
\hline Desviación típica & $6.532,80$ & $11.647,00$ & $13.275,00$ & $6.463,80$ & $1.998,90$ & $2.258,20$ & $9.623,10$ & $3.711,20$ \\
\hline C.V. & 0,27 & 0,25 & 0,61 & 0,40 & 0,40 & 1,20 & 1,09 & 0,43 \\
\hline Asimetría & 0,76 & 0,86 & 0,61 & 1,58 & 0,39 & 0,87 & 0,43 & 1,20 \\
\hline Exc. de curtosis & 1,45 & 3,55 & $-0,37$ & 4,80 & $-0,76$ & $-0,38$ & $-1,39$ & 1,22 \\
\hline
\end{tabular}

En cuanto a las búsquedas que se realizan en Google sobre estos museos también tiene niveles muy dispares ya sea atendiendo al total de búsquedas en el periodo analizado (véase figura 3) como si comparamos las series temporales de búsquedas realizadas para cada museo (véase figura 4). 
Figura 3

\section{BÚSQUEDA EN INTERNET}

Totales

museo del prado

museo reina sofia

museo de altamira

museo arqueológico... ।

museo casa de amér...
(2)

Figura 4

SERIES TEMPORALES DE BÚSQUEDAS EN CADA MUSEO

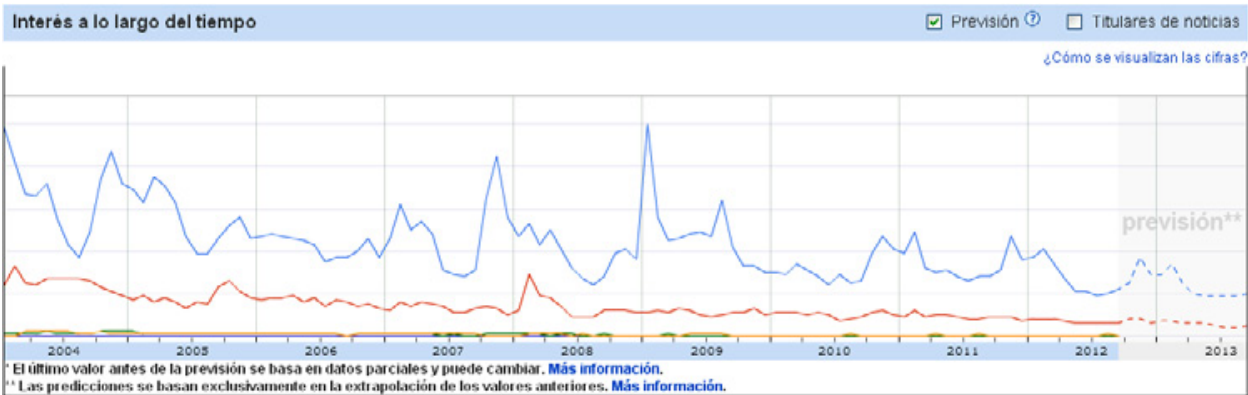

Figura 5

USUARIOS DE INTERNET EN RELACIÓN A LA POBLACIÓN

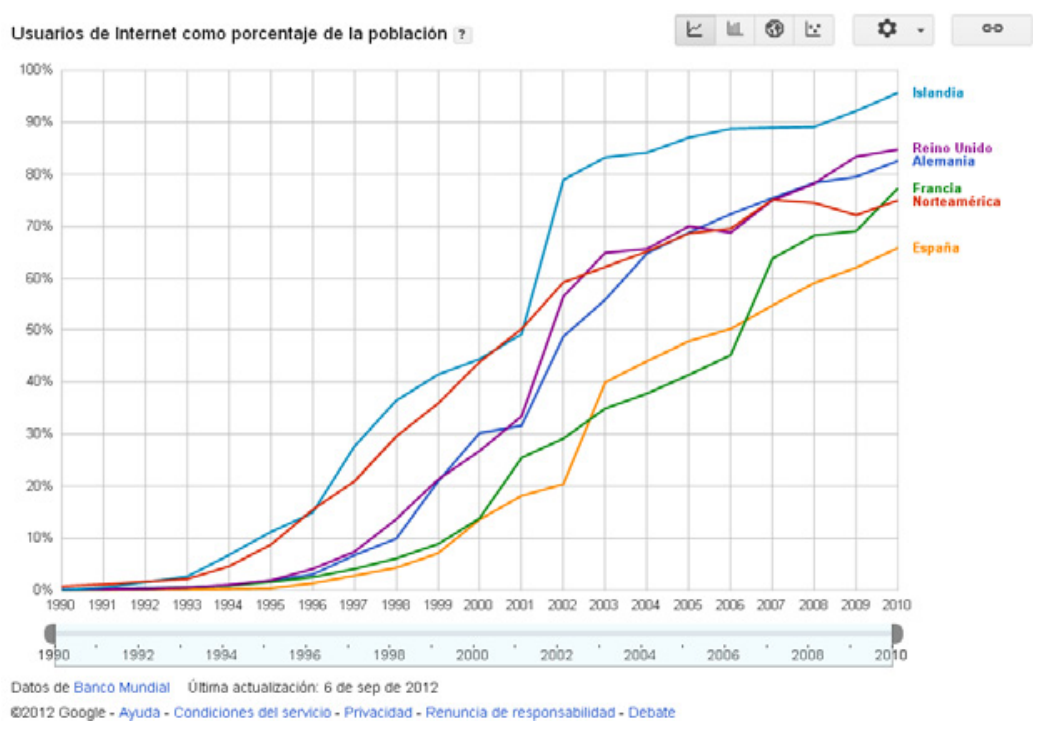

Cuadernos de Turismo, 38, (2016), 203-219 
Estas búsquedas de Google no se han filtrado ni por tipo de búsqueda, ni por zona geográfica ni por área de interés con el objetivo de mostrar el interés global por cada museo. Consideramos que el periodo analizado es suficiente ya que trabajamos con más de 400 observaciones para muestras semanales y entorno a 100 observaciones en frecuencias mensuales, lo que consideramos un tamaño muestral adecuado.

Así mismo pensamos que el uso de Internet desde el año 2004 es relevante ya que, como se puede observar en la figura 5, desde ese año los niveles de acceso a Internet rondan el $70 \%$ en los países desarrollados y el crecimiento en el acceso a Internet empieza a ser decreciente.

\section{RESULTADOS}

Para trabajar con más facilidad y aproximarnos a los supuestos que exige el Modelo Lineal General hacemos dos transformaciones habituales en la técnica econométrica y trabajamos con la diferencia logarítmica de visitantes y búsquedas en Google, así como con la diferencia estacional. Los resultados de las regresiones realizadas están resumidos en la tabla 4.

Tabla 4

\section{REGRESIONES}

\begin{tabular}{|c|c|c|c|c|c|c|c|c|c|c|c|c|}
\hline & \multicolumn{3}{|c|}{ MCO log diferencias } & \multicolumn{3}{|c|}{$\begin{array}{c}\text { MCO diferencias } \\
\text { estacionales }\end{array}$} & \multicolumn{3}{|c|}{ MCG log diferencias } & \multicolumn{3}{|c|}{$\begin{array}{c}\text { MCG diferencias } \\
\text { estacionales }\end{array}$} \\
\hline & $\beta$ & t & p-value & $\beta$ & $\mathbf{t}$ & p-value & $\beta$ & t & p-value & $\beta$ & $\mathrm{t}$ & p-value \\
\hline $\begin{array}{l}\text { Museo Reina } \\
\text { Sofía }\end{array}$ & 0,32 & 6,33 & 0,00 & 262,35 & 12,79 & 0,00 & 0,39 & 7,33 & 0,00 & 241,13 & 10,35 & 0,00 \\
\hline $\begin{array}{l}\text { Museo del } \\
\text { Prado } \\
\end{array}$ & 0,15 & 3,34 & 0,00 & 452,64 & 7,54 & 0,00 & 0,14 & 3,05 & 0,00 & 210,30 & 3,29 & 0,00 \\
\hline $\begin{array}{l}\text { Museo de } \\
\text { Altamira }\end{array}$ & 0,45 & 3,32 & 0,00 & $-6,04$ & $-0,27$ & 0,78 & 0,32 & 2,78 & 0,01 & $-3,32$ & $-0,16$ & $\mathbf{0 , 8 8}$ \\
\hline $\begin{array}{l}\text { Museo } \\
\text { Arqueológico } \\
\text { Nacional }\end{array}$ & 0,01 & 0,44 & 0,65 & 1,98 & 0,03 & 0,97 & 0,15 & 1,01 & 0,32 & 21,56 & 0,38 & 0,71 \\
\hline $\begin{array}{l}\text { Museo Casa } \\
\text { de América }\end{array}$ & 0,28 & 2,54 & 0,01 & 24,75 & 3,02 & 0,00 & n.a. & n.a. & n.a. & $-7,50$ & $-0,76$ & 0,45 \\
\hline $\begin{array}{l}\text { Museo Casa } \\
\text { Cervantes } \\
\end{array}$ & 0,29 & 1,32 & $\mathbf{0 , 2 0}$ & 56,59 & 7,18 & 0,00 & n.a. & n.a. & n.a. & 12,25 & 2,57 & 0,01 \\
\hline $\begin{array}{l}\text { Museo del } \\
\text { Greco }\end{array}$ & $-0,05$ & $-0,21$ & 0,84 & 252,81 & 3,83 & 0,00 & n.a. & n.a. & n.a. & 11,80 & 0,38 & 0,70 \\
\hline $\begin{array}{l}\text { Museo } \\
\text { Nacional de } \\
\text { Escultura }\end{array}$ & 0,10 & 0,68 & $\mathbf{0 , 5 0}$ & $-1,46$ & $-0,13$ & 0,90 & 0,20 & 1,46 & 0,15 & 0,13 & 0,01 & 0,99 \\
\hline
\end{tabular}

Observamos que los parámetros $\beta$ calculados por Mínimos Cuadrados Ordinarios (MCO) para los dos museos de mayor volumen de visitantes (Reina Sofía y Prado) son positivos y significativos al 99\%, lo que nos llevaría a validar las dos hipótesis que hemos planteado en este estudio. No obstante, tal y como se puede observar en los listados de 
las regresiones realizadas contenidos en el Anexo, debido a la simplicidad del modelo la capacidad explicativa según el parámetro $\mathrm{R}^{2}$ es muy baja, y no se superan los contrastes de normalidad en residuos, ausencia de autocorrelación y heteroscedasticidad. Esto implica que los contrates de significatividad individual del estadístico «t» deben ser interpretados con cautela ya que estas estimaciones aunque siguen siendo lineales e insesgadas no son óptimas. Para salvar estas carencias en la especificación del modelo realizamos la misma regresión por Mínimos Cuadrados Generalizados (MCG) utilizando el algoritmo de Cochrane-Orcutt llegando a las mismas conclusiones.

A medida que vamos trabajando con museos de un volumen de asistencia menor observamos que, aunque se mantiene el signo positivo en los parámetros calculados, su nivel de significatividad es menor, tanto por MCO como por MCG.

La interpretación de los parámetros contenidos en la tabla 4 nos lleva a las siguientes conclusiones:

- Validamos H1 para museos grandes: Las series históricas de estadísticas de búsquedas en Google son una variable con capacidad para explicar los niveles de asistencia a museos populares con altos niveles de asistencia, siendo su capacidad explicativa limitada para museos pequeños.

- Validamos H2: Un mayor interés por un museo implica un mayor volumen de visitantes.

Consideramos es estudio puede significar la aparición de una nueva variable, fácil de obtener, con capacidad explicativa a la hora de gestionar una institución cultural.

\section{CONCLUSIONES}

La importancia del turismo cultural en España está incrementándose en los últimos años como consecuencia de un cúmulo de factores como es la aparición de fuertes competidores y la alta estacionalidad. Pero también debe reconocerse el esfuerzo realizado por las Administraciones Turísticas y las empresas interesadas para potenciar este tipo de turismo y así aprovechar las ventajas que ofrece, como es la desestacionalidad y el incremento de los ingresos que produce.

Las acciones desarrolladas para potenciar este tipo de turismo han sido llevadas a cabo por las entidades públicas competentes y por algunas empresas privadas. Entre estas últimas se encuentra los museos, que son centros donde se pueden exponer obras de arte a la vez que permiten conservarlas y restaurarlas. Estos lugares hacen una labor de comunicación importante tanto para residentes como para turistas, informando por ejemplo de sus colecciones permanentes así como de aquellas temporales que exponen.

Para determinar el proceso de decisión de compra de los consumidores de los museos se ha utilizado el propuesto por los profesores Blackwell, Miniard y Engel (2005) que identifica cinco etapas en este proceso. Se inicia con el reconocimiento de la necesidad, en este caso de la necesidad de visitar un museo como consecuencia de diversos motivos como puede ser el aprendizaje y la educación. Una vez detectada la necesidad el consumidor procede a buscar información, para lo cual comienza con un análisis interno haciendo 
uso de la memoria y que posteriormente concluye con una búsqueda externa que va desde la opinión de amigos o familiares hasta la utilización de medios masivos o below the line. Con toda la información recibida a través de los distintos medios utilizados, el consumidor procede a evaluar las alternativas que tiene y así compara entre sus expectativas y la oferta para poder hacer una elección final y así decidir qué museo va a visitar finalmente o porque colección se decanta. Con todo esto se encuentra con posibilidad de pasar a la etapa siguiente que sería la conducta post compra.

De este proceso de decisión de compra ha habido una etapa que ha sufrido grandes cambios en los últimos años como consecuencia del desarrollo de las tecnologías y su incorporación como un sistema de búsqueda de información de uso generalizado entre los consumidores. De este modo, la herramienta de Internet viene incrementando su importancia anualmente entre los medios masivos de información. De igual manera, que los museos más importantes también están incrementando su presencia en este medio llegando incluso a permitir visitar sus exposiciones a través de Internet.

Teniendo en cuenta todas estas circunstancias expuestas, se planteó la posibilidad de que los visitantes realizarán búsquedas en Internet que permitieran conocer la futura asistencia de los consumidores a esos museos por los que se interesaban. Con este trabajo tratábamos de averiguar si a través de las series históricas de estadísticas de un motor de búsqueda, en concreto Google, se tendría capacidad suficiente para predecir la afluencia de público a los museos. Nuestro estudio demuestra que las estadísticas de búsqueda tienen cierta capacidad explicativa sobre la asistencia a los museos, siendo esta capacidad explicativa mayor en los museos populares con mayores niveles de asistencia que en los museos pequeños. Además, se ha podido confirmar que un mayor interés por un determinado museo, esto es, un mayor número de búsquedas en Google de un museo concreto, supone una mayor afluencia de visitantes.

\section{BIBLIOGRAFÍA}

ASKITAS, N. y ZIMMERMANN, K. (2009): «Google Econometrics and Unemployment Forecasting». DIW Berlin Discussion Paper, $\mathrm{n}^{\circ}$ 899, http://ssrn.com/abstract=1465341 ó http://dx.doi.org/10.2139/ssrn.1465341 (visitado en Junio 2013)

BLACKWELL, R. D., MINIARD, P.W. y ENGEL, J.F. (2005): Consumer Behavior, South-Western College Pub, $10^{\mathrm{a}}$ ed.

BOLLEN, J., MAO, H. y ZENG, X. (2010): Twitter mood predicts the stock market. http:// hughchristensen.co.uk/papers/socialNetworking/1010.3003v1.pdf

BOURDIEU, P., DARBEL, A. y SCHNAPPER, D. (1997): The Love of Art: European Art Museums and their Public, Polity Press.

CARE. (2009): http://www.care-aam.org/default.aspx Retrieved on 08.09.09.

DEL BARRIO, M.J., HERRERO, L.C. y SANZ, J.A. (2009): «Measuring the efficiency of heritage institutions: A case study of a regional system of museums in Spain». Journal of Cultural Heritage, $\mathrm{n}^{\circ} 10$, pp. 258-268.

DIMAGGIO, P. (1996): «Are art-museum visitors different from other people? The relationship between attendance and social and political attitudes in the United States». Poetics, vol. 24, pp. 161-180. 
DIMAGGIO, P., USEEM, M. y BROWN, P. (1978): «Audience Studies of the Performing Arts, and Museums: A Critical Review». National Endowment of the Arts, $\mathrm{n}^{\circ} 9$.

FALK, J.H. y DIERKING, L.D. (2000): Learning from Museums: Visitor Experiences and the Making of Meaning. Altamira Press.

FORD, M. (1992): Motivating humans: Goals, emotions and personal agency beliefs, Newbury Park, CA: Sage.

GANZEBOOM, H.B.G. (1982): «Explaining differential participation in high-cultural activities: A confrontation of information-processing and status seeking theories.». In Werner, R. (1982): Theoretical Models and Empirical Analyses, pp. 186-205, Utrecht: E.S. Publications.

GARCÍA, A. (2002): «¿Usuarios o visitantes de museos?». Museos, nº 6, pp. 1-18.

GEROW, A. y KEANE, M.T. (2011): Mining the Web for the «Voice of the Herd» to Spot Stock Market Bubbles, International Joint Conference of Artificial Inteligence.

GÓMEZ, R. (2012): El préstamo de valores en España: Relevancia de la venta en corto y el estado de ánimo de los inversores en la rentabilidad de la Bolsa Española. Editorial Académica Española, Madrid.

GÓMEZ, R. (2013): «Señales de inversión basadas en un índice de aversión al riesgo», Investigaciones Europeas de Dirección y Economía de la Empresa, nº 19, vol. 3, pp. 147-157.

GÓMEZ, R. y PRADO, C. (2013): «¿Dónde podemos ver lo que votamos los españoles?», XXVII Congreso Anual de AEDEM. Barcelona.

HARRISON, J. (1997): «Museums and touristic expectations». Annals of Tourism Research, vol. 24, $\mathrm{n}^{\circ}$ 1, pp. 23-40.

HOLLENBECK, C.R., PETERS, C. y ZINKHAN, G.M. (2008): «Retail spectacles and brand meaning: Insights from a brand museum case study». Journal of Retailing, vol. 84, no 3, pp. 334-353.

HOOD, M.G. (1983): «Staying Away: Why people choose not to visit museums». Museum News, vol. 61, no 4, pp. 50-57.

HOWARD FALK, J.J. y DIERKING, LD. (2000): «The Museum Experience». In B. Sheppard (Ed.), Perspectives on outcome based evaluation for libraries and museums (pp.4e12). Washington, D.C.: Institute of Museum and Library Services.

INSTITUTO DE ESTUDIOS TURÍSTICOS (IET) (2014a): Movimientos Turísticos en Fronteras (Frontur) 2013, Instituto de Estudios Turísticos, www.iet.tourspain.es (visitado en Enero de 2014).

INSTITUTO DE ESTUDIOS TURÍSTICOS (IET) (2014b): Encuesta de Gasto Turístico (Egatur) 2013, Instituto de Estudios Turísticos, www.iet.tourspain.es (visitado en Enero de 2014).

INSTITUTO DE ESTUDIOS TURÍSTICOS (IET) (2014c): Visitantes del Museo del Prado, Instituto de Estudios Turísticos, www.iet.tourspain.es (visitado en Enero de 2014).

KOTLER, P., BOWEN, J. y MAKENS, J. (2004): Marketing Turístico, Pearson Educación, Madrid, España, $3^{\mathrm{a}} \mathrm{Ed}$.

MCLAREN, N. y SHANBHIDGE, R. (2011): Using Internet Search Data as Economic Indicators, Bank of England Quarterly Bulletin http://www.bankofengland.co.uk/publications/quarterlybulletin/qb110206.pdf (visitado en Junio 2013). 
MINISTERIO DE EDUCACIÓN, CULTURA Y DEPORTE (2014): Cifras de los visitantes de los Museos Estatales, http://www.mcu.es/visitantemuseo/cargarFiltroBusqueda. do?layout=visitantemuseo\&cache=init\&language=es (visitado en enero 2014).

ORTEGA, E. y RODRÍGUEZ, B. (2008): «El turismo cultural en el marco del turismo social en España», en Troitiño, M.A., García, J.S. y García, M. (2008): Destinos Turísticos: Viejos Problemas ¿Nuevas Soluciones?, X Congreso de Geografía del Turismo, Ocio y Recreación, (A.G.E.), Ediciones de la Universidad de Castilla La Mancha, Cuenca, pp. 295-310.

PACKER, J. y BALLANTYNE, R. (2002): «Motivational factors and the visitor experience: A comparison of three sites». Curator, vol. 45, n 3, pp. 183-198.

PRENTICE, R.; DAVIES, A. y BEEHO, A. (1997): «Seeking generic motivations for visiting and not visiting museums and like cultural attractions». Museum Management and Curatorship, vol. 16, $\mathrm{n}^{\circ} 1$, pp. 45-70.

ROMPF, P.D. (2002): «Comparative studies on gratuitous referrals: some cross-cultural connotations». Frontiers in Southeast I-CHRIE, vol. 6, n ${ }^{\circ}$ 1, pp. 1-3.

ROMPF, P.D. y RICCI, P. (2005): «Gratuitous referrals: a subset of visitor search activities and decision strategies at destination for travel-related services». Journal of Travel and Tourism Marketing, vol. 18, n 2, pp. 125-142.

ROSE, A.K. y SPIEGEL, M.M. (2012): «Dollar illiquidity and central bank swap arrangements during the global financial crisis». Journal of International Economics, vol. $88, n^{\circ} 2$, pp. 326-340.

SCHLIEWEN, R.E. (1977): A leisure study -Canada 1975. Arts and Culture Branch, Department of the Secretary of State, Ottawa, Canada.

SCHUSTER, J. (2001): Policy and Planning with a Purpose or the Art of Making Choices in Arts Funding, In The Third Arts Plan: Policy and Planning with a Purpose, Dublin, Irlanda.

SCHUSTER, J.M.D. (1991): The audience for American art museums National Endowment for the Arts, Research Division Report 23, Seven Locks Press, Washington, DC.

SGT (2008): Plan del Turismo Español Horizonte 2020. Documento ejecutivo, Secretaría General de Turismo, Ministerio de Industria, Turismo y Comercio, hppt://www. turismo2020.es/index.php?/esp/documentación (visitado en octubre 2013).

SHENG, C.W. y CHEN, M.C. (2012): «A study of experience expectations of museum visitors». Tourism Management, vol. 33, pp. 53-60.

SMITH, G.P. (2012): «Google Internet search activity and volatility prediction in the market for foreign currency», Finance Research Letters, vol. 9, n 2, pp. 103-110.

SOCIALWIN (2014): Presentación del primer informe de redes sociales y museos 2013, Socialwin y Museus Nacional D’Art de Catalunya, Barcelona, España.

STYLIANOU, T. y CYPRUS, L. (2010): «Gazing from home: cultural tourism and art museums». Annals of Tourism Research, vol. 38, n 2, pp. 403-421.

VLASTAKIS, N. y MARKELLOS, R.N. (2012): « Information demand and stock market volatility». Journal of Banking \& Finance, vol. 36, nº 6, pp. 1.808-1.821. 
